\title{
Model-free Predictive Control
}

\author{
Anders Stenman \\ Department of Electrical Engineering \\ Linköping University, S-581 83 Linköping, Sweden \\ WWW: http://wWw. control.isy.liu.se \\ Email: stenman@isy.liu.se
}

February 25, 1999

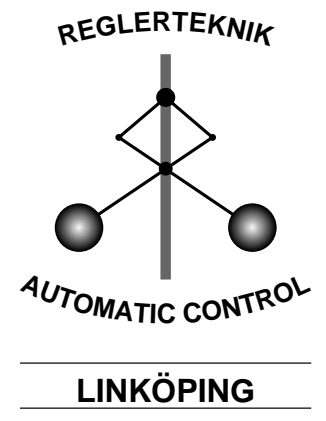

Report no.: LiTH-ISY-R-2119

Submitted to CDC '99

Technical reports from the Automatic Control group in Linköping are available by anonymous ftp at the address ftp.control.isy.liu. se. This report is contained in the compressed postscript file 2119.ps.Z. 


\title{
Model-free Predictive Control
}

\author{
Anders Stenman \\ Department of Electrical Engineering, \\ Linköping University, SE-581 83 Linköping, Sweden. \\ Phone: +461328 40 79, Fax: +4613282622 \\ Email: stenman@isy.liu.se
}

February 25, 1999

\begin{abstract}
Model predictive control, MPC, form a class of model-based controllers that select control actions by on-line optimization of objective functions. Design methods based on MPC have found wide acceptance in industrial process control applications, and have been thoroughly studied by the academia. Most of the work so far have relied on linear models of different sophistication because of their advantage of providing simple and straightforward implementations. However, when turning to the nonlinear domain, problems often arise as a consequence of the difficulties in obtaining good nonlinear models, and the computational burden associated with the control optimization. In this paper we present a new approach to the nonlinear MPC problem using the recently proposed concept of model-on-demand. The idea is to estimate the process dynamics locally and on-line using process data stored in a database. By treating the local model obtained at each sample time as a local linearization, it is thus possible to reuse tools and concepts from the linear MPC framework. Three different variants of the idea, based on local linearization, linearization along a trajectory and nonlinear optimization respectively, are studied. They are all illustrated in numerical simulations.
\end{abstract}

Keywords: predictive control, local polynomial models

\section{Introduction}

Model predictive control, MPC, is a family of optimal-control related methods that selects control actions by on-line minimization of objective functions. The methods assume a model of the controlled process and utilize its predictive power to optimize the control.

There are many variants of model predictive control, for instance, dynamic matrix control and extended horizon control. See García et al. (1989) for a survey. If the model is estimated on-line in an adaptive control manner, one usually talks about generalized predictive control, GPC, (Clarke et al., 1987a,b).

Early formulations of MPC used linear models of various sophistication such as step response, im- pulse response or state-space models, and turned out to be successful in controlling linear and mildly nonlinear processes. The performance degradation and instability noticed in the presence of strong nonlinearities, though, soon motivated the extension to nonlinear models. However, both the difficulties in obtaining a good model of the nonlinear process and the excessive computational burden associated with the control optimization have been serious obstacles to widespread industrial implementations. Various simplifications and approximations based on linearization of the nonlinear process have therefore been proposed, see, for instance, Gattu and Zafiriou (1992). In this paper we instead present the concept of model-free predictive control, which extends the MPC ideas to the model-on-demand framework.

The model-on-demand philosophy has its origins in local polynomial modeling and has been brought up as an alternative to more traditional modeling methods like neural nets, radial basis networks and wavelets, and has for functional approximations been extensively studied within the statistical literature (see, for instance, Cleveland and Devlin (1988), Wand and Jones (1995), or Fan and Gijbels (1996)). Rather than estimating a large global model covering the entire regressor space, the idea is instead to model the input-output data belonging to a small neighborhood around the current operating point (Stenman et al., 1996; Stenman, 1997). This gives the advantage of providing a good fit using a significantly simpler model than the one needed for a good global approximation.

For simplicity and ease of notation we will in this paper restrict ourselves to SISO processes of NARX type, That is, systems of the form

$$
y(t)=m(\varphi(t))+e(t), \quad t=1, \ldots, M,
$$

where $\varphi(t)$ denotes a regression vector which con- 
sist of lagged input-output data, $m(\cdot)$ is an unknown nonlinear mapping and $e(t)$ is a noise term modeled as i.i.d. random variables with zero means and variances $\sigma_{t}^{2}$.

The organization of the paper is as follows: Section 2 describes the basics behind modeling-ondemand, Section 3 describes the MPC setup and discusses how local modeling can be incorporated in this framework. Section 4 illustrates the proposed methods in numerical simulations, and Section 5, finally, provides some concluding remarks.

\section{Model-on-Demand}

The basic idea behind the model-on-demand philosophy is to store all observations $\{(y(k), \varphi(k)\}$ from the process in a database, and estimate the system dynamics locally and "on demand" when the need for a model arises. That is, for each operating point $\varphi(t)$, a local model is obtained via the weighted regression problem;

$$
\begin{aligned}
\hat{\beta}=\underset{\beta}{\arg \min } \sum_{k=1}^{M} & \ell(y(k)-m(\varphi(k), \beta)) \\
& \times W\left(\frac{\|\varphi(k)-\varphi(t)\|_{\mathbf{M}}}{h}\right),
\end{aligned}
$$

where $\ell(\cdot)$ is a scalar-valued and positive norm function, $\|u\|_{\mathbf{M}} \triangleq \sqrt{u^{T} \mathbf{M} u}$ is a scaled distance function (vector norm) on the regressor space, $h$ is a bandwidth parameter controlling the size of the neighborhood around $\varphi(t)$, and $W(\cdot)$ is a window function (usually referred to as the kernel) assigning weights to each remote data point according to its distance from $\varphi(t)$. The window is typically a bellshaped function with bounded support. See Figure 1 , where some commonly used windows are depicted.

In principle, it is possible to use any nonlinear model structure as local model in (2). However, if the quadratic $L_{2}$ norm, $\ell(\varepsilon)=\varepsilon^{2}$, is used and the model is linear in the unknown parameters, the estimate can be easily computed using simple and powerful weighted least squares. We thus assume a local linear model structure,

$$
m(\varphi(k), \beta)=\beta_{0}+\beta_{1}^{T}(\varphi(k)-\varphi(t))
$$

as the the default choice in the sequel of the paper. If $\hat{\beta}_{0}$ and $\hat{\beta}_{1}$ denote the minimizers of (2) using the model (3), it is easy to see that an estimate of $y(t)$ (i.e., a one-step-ahead prediction), is given by

$$
\hat{y}(t)=m(\varphi(t), \hat{\beta})=\hat{\beta}_{0} .
$$

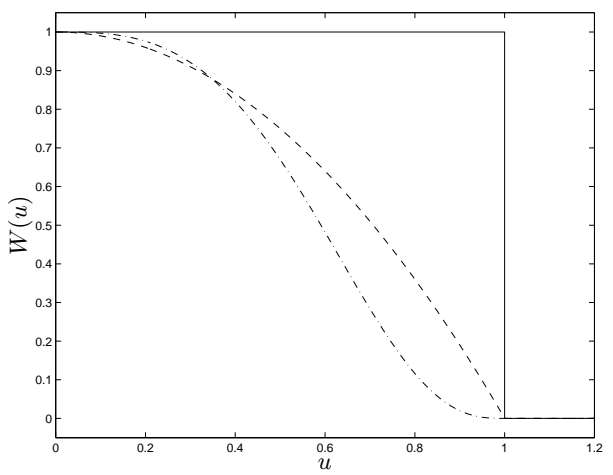

Figure 1: Some commonly used window functions: Uniform (solid), Epanechnikov, $W(u)=\left(1-u^{2}\right)_{+}$ (dashed), and tricube, $W(u)=\left(1-u^{3}\right)_{+}^{3}$ (dashdotted).

However, it has been shown that it also is possible to enhance the estimate by first estimating higher order derivatives of $m(\cdot)$ from data, and plugging them into an additional optimization step. See Stenman (1997) and Stenman et al. (1997) for more details around this.

Note that the formulation (2) produces a single local estimate $\hat{y}(t)$ associated with the current regression vector $\varphi(t)$. To obtain predictions at other locations in the regressor space, the weights change and new optimization problems have to be solved. This is in contrast to the global modeling approach where the model is fitted to data only once. However, in a neighborhood around $\varphi(t)$, the local linear model (3) provides an input-output linearization of the form

$$
A\left(q^{-1}\right) y(t)=B\left(q^{-1}\right) u\left(t-n_{k}\right)+\alpha,
$$

where $A\left(q^{-1}\right)$ and $B\left(q^{-1}\right)$ are polynomials in the backward time-shift operator $q^{-1}$ obtained from the components of $\hat{\beta}_{1}$, and

$$
\alpha=\hat{\beta}_{0}-\hat{\beta}_{1}^{T} \varphi(t) .
$$

is an offset term.

It is well known that the bandwidth $h$ has a critical impact on the quality of the estimate (4), since it governs a trade-off between the bias and variance errors. Methods that use the available data to produce good bandwidths are usually referred to as (data-driven) bandwidth selectors, and have been thoroughly studied within the statistical literature, see, for instance, Wand and Jones (1995) or Fan and Gijbels (1996). They can roughly be divided into "classical" methods which are based 
on cross-validation ideas, and "plug-in" methods which rely on minimizing asymptotic MSE expressions (Loader, 1995). The majority of the bandwidth selectors proposed so far, have been of global type, i.e., they produce a single global value. However, adaptive (i.e., local) methods, that select bandwidths on-line for each estimation point, have gained a significant interest in recent years, although the development of them still seems to be an open and active research area. The bandwidth selection problem is outside the scope of this paper, though, and in the applications that follow we will not pay it any deeper attention.

The scaling matrix $\mathbf{M}$ that controls the distance function (and hence the shape of the neighborhood) can be optimized in a similar way, although we here only have considered fixed choices. Selecting the scaling matrix is very important, though, especially when the regressor components have very different magnitudes. An obvious default choice, which has been adopted here, is to make it proportional to the inverse covariance of the regressors.

\section{Model Predictive Control}

The model predictive control problem can be formulated as follows (Meadows and Rawlings, 1997): Given a model description of the form (1) and knowledge of the current system state, seek a control that minimizes the objective function

$$
\begin{aligned}
J= & \sum_{k=0}^{N-1} Q_{e}(k)(r(t+k+1)-\hat{y}(t+k+1))^{2} \\
& +Q_{u}(k) u^{2}(t+k)+Q_{\Delta u}(k) \Delta u^{2}(t+k)
\end{aligned}
$$

where $Q_{e}(k), Q_{u}(k)$ and $Q_{\Delta u}(k)$ represent penalties on the control error, control signal and control increment magnitudes respectively. Of the $N$ future control actions that minimize $J$, only the first one is applied to the controlled process. When new measurements become available, a new optimization problem is formulated whose solution provides the next control action. This is usually referred to as the receding horizon principle. Another special feature of the formulation (7) is the presence of the control increment,

$$
\Delta u(t+k)=u(t+k)-u(t+k-1),
$$

in the objective. In some examples, for instance in process control applications, the change rate of the control action may be restricted. Rather than including the actuator dynamics in the model, it is instead a common practice to include penalties on the control increment. An additional advantage with MPC is that it is straightforward to include hard bounds on the control signal magnitude and the control increment, i.e.,

$$
\begin{gathered}
u_{\min } \leq u(t+k) \leq u_{\max } \\
\Delta u_{\min } \leq \Delta u(t+k) \leq \Delta u_{\max }
\end{gathered}
$$

Optimization of (7) can be quite demanding for large prediction horizons. To decrease the computational complexity it is thus very common to introduce constraints on the future control signals. An often used approach is to assume that the control increments are zero after $N_{u} \leq N$ steps;

$$
\Delta u(t+k-1)=0, \quad k>N_{u} .
$$

It is well known that this also has the effect of producing less aggressive controllers (Meadows and Rawlings, 1997). The quantity $N_{u}$ is usually referred to as the control horizon.

\subsection{Optimization Based on Local Linearizations}

The most obvious way of incorporating the modelon-demand approach into the MPC formulation is to optimize the objective (7) based on the local model obtained at time $t$. A quite similar idea was explored by Gattu and Zafiriou (1992) using statespace models, but here we choose to remain in the input-output domain.

From the model (3) we obtain an input-output linearization of the form (5). The basic problem is now to express the output prediction at time $t+k$ as a function of future controls. The standard trick (Åström and Wittenmark, 1995) is to introduce the identity

$$
1=A\left(q^{-1}\right) F_{k}\left(q^{-1}\right)+q^{-k} G_{k}\left(q^{-1}\right)
$$

where $F_{k}\left(q^{-1}\right)$ and $G_{k}\left(q^{-1}\right)$ are polynomials of degrees $k-1$ and $n_{a}-1$ respectively. Substituting (11) into (5) yields

$$
\begin{aligned}
y(t)=B\left(q^{-1}\right) & F_{k}\left(q^{-1}\right) u(t-1) \\
& +q^{-k} G_{k}\left(q^{-1}\right) y(t)+F_{k}(1) \alpha,
\end{aligned}
$$

i.e.,

$$
\begin{aligned}
\hat{y}(t+k)=B\left(q^{-1}\right) & F_{k}\left(q^{-1}\right) u(t+k-1) \\
& +G_{k}\left(q^{-1}\right) y(t)+F_{k}(1) \alpha .
\end{aligned}
$$

By partitioning $B\left(q^{-1}\right) F_{k}\left(q^{-1}\right)$ as

$$
B\left(q^{-1}\right) F_{k}\left(q^{-1}\right)=S_{k}\left(q^{-1}\right)+q^{-k} \tilde{S}_{k}\left(q^{-1}\right),
$$


where $\operatorname{deg} S_{k}\left(q^{-1}\right)=k-1$ and $\operatorname{deg} \tilde{S}_{k}\left(q^{-1}\right)=n_{b}-$ 2 , the output prediction (13) can be rewritten as

$$
\hat{y}(t+k)=S_{k}\left(q^{-1}\right) u(t+k-1)+\bar{y}(t+k) .
$$

Here the first term depends on future control actions $u(t), \ldots, u(t+k-1)$ whereas the remaining terms (collected into $\bar{y}(t+k)$ ) depend on measured quantities only. By introducing the notations

$$
\begin{aligned}
& \hat{\mathbf{y}} \triangleq\left(\begin{array}{lll}
\hat{y}(t+1) \quad \ldots \quad \hat{y}(t+N))^{T},
\end{array}\right. \\
& \tilde{\mathbf{u}} \triangleq\left(\begin{array}{lll}
u(t) \quad \ldots \quad u(t+N-1))^{T},
\end{array}\right.
\end{aligned}
$$

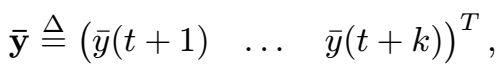

$$
\begin{aligned}
& \tilde{\mathbf{S}} \triangleq\left(\begin{array}{cccc}
s_{0} & 0 & \ldots & 0 \\
s_{1} & s_{0} & \ldots & 0 \\
\vdots & & \ddots & \vdots \\
s_{N-1} & s_{N-2} & \ldots & s_{0}
\end{array}\right),
\end{aligned}
$$

where $s_{i}$ are the coefficients of $S_{k}\left(q^{-1}\right)$ we have that

$$
\hat{\mathbf{y}}=\overline{\mathbf{y}}+\tilde{\mathbf{S}} \tilde{\mathbf{u}} \text {. }
$$

However, taking into account that the control horizon $N_{u}$ typically is shorter than the prediction horizon $N$ and that (10) holds, this can be rewritten as

$$
\hat{\mathbf{y}}=\overline{\mathbf{y}}+\mathbf{S u},
$$

where

$$
\mathbf{u} \triangleq\left(u(t) \quad \ldots \quad u\left(t+N_{u}-1\right)\right)^{T},
$$

and

$$
\mathbf{S} \triangleq \tilde{\mathbf{S}} \boldsymbol{\Lambda} \text { with } \boldsymbol{\Lambda} \triangleq\left(\begin{array}{cccccc}
1 & 0 & \ldots & 0 & \ldots & 0 \\
0 & 1 & \ldots & 0 & \ldots & 0 \\
\vdots & \vdots & \ddots & \vdots & & \vdots \\
0 & 0 & \ldots & 1 & \ldots & 1
\end{array}\right)^{T}
$$

The control increments (8) can also be expressed in vector form

$$
\Delta \mathbf{u}=\mathbf{D u}-\overline{\mathbf{u}}
$$

by introducing the auxiliary quantities

$\mathbf{D} \triangleq\left(\begin{array}{cccc}1 & 0 & \ldots & 0 \\ -1 & 1 & & \vdots \\ 0 & \ddots & \ddots & 0 \\ 0 & \ldots & -1 & 1\end{array}\right)$ and $\overline{\mathbf{u}} \triangleq\left(\begin{array}{c}u(t-1) \\ 0 \\ \vdots \\ 0\end{array}\right)$

The objective (7) can thus be simplified as

$$
\begin{aligned}
& J(\mathbf{u})=\|\mathbf{r}-\hat{\mathbf{y}}\|_{\mathbf{Q}_{\mathbf{e}}}^{2}+\|\mathbf{u}\|_{\mathbf{Q}_{\mathbf{u}}}^{2}+\|\boldsymbol{\Delta} \mathbf{u}\|_{\mathbf{Q}_{\Delta \mathbf{u}}}^{2} \\
& =\|\mathbf{r}-\overline{\mathbf{y}}-\mathbf{S u}\|_{\mathbf{Q}_{\mathbf{e}}}^{2}+\|\mathbf{u}\|_{\mathbf{Q}_{\mathbf{u}}}^{2}+\|\mathbf{D u}-\overline{\mathbf{u}}\|_{\mathbf{Q}_{\mathbf{\Delta} \mathbf{u}}}^{2},
\end{aligned}
$$

where

$$
\mathbf{r} \triangleq(r(t+1) \quad \ldots \quad r(t+N))
$$

denotes the desired (and possibly smoothed) reference trajectory, and $\mathbf{Q}_{\mathbf{e}}, \mathbf{Q}_{\mathbf{u}}$ and $\mathbf{Q}_{\boldsymbol{\Delta} \mathbf{u}}$ are diagonal matrices with entries $Q_{e}(k), Q_{u}(k)$ and $Q_{\Delta u}(k)$ respectively. For the unconstrained case, the minimizing control sequence is thus obtained explicitly by means of ordinary least squares theory.

For the constrained case, the constraints (9) can be re-formulated as

$$
\mathrm{Cu} \leq \mathbf{c}
$$

where

$$
\mathbf{C} \triangleq\left(\begin{array}{c}
I \\
-I \\
\mathbf{D} \\
-\mathbf{D}
\end{array}\right) \text { and } \mathbf{c} \triangleq\left(\begin{array}{c}
u_{\max } \cdot \mathbf{1} \\
-u_{\min } \cdot \mathbf{1} \\
\Delta u_{\max } \cdot \mathbf{1}+\overline{\mathbf{u}} \\
-\Delta u_{\min } \cdot \mathbf{1}-\overline{\mathbf{u}}
\end{array}\right)
$$

We thus obtain a quadratic programming (QP) problem which can be efficiently solved using standard numerical optimization software.

\subsection{Optimization Based on Lin- earization along a Trajectory}

A drawback with the previously described approach is that the predicted output behavior of the process is based on the input-output linearization obtained at time $t$. This assumption will not hold in general, since when the currently computed control is applied to the process, the operating point will most likely change. A natural solution to this problem is to provide the optimized control sequence $\mathbf{u}$ obtained from the previous optimization to the local estimator, in order to obtain an approximate timevarying local linear model over the future $N$ samples. A similar approach was formulated by Löfberg (1998) using state-space models.

The local estimator will at each time instant $\tau=$ $t+1, \ldots, t+N$ return a input-output linearization of the form

$$
A_{\tau}\left(q^{-1}\right) y(\tau)=B_{\tau}\left(q^{-1}\right) u(\tau)+\alpha_{\tau}
$$

The output predictor associated with this timevarying model can be derived similar to the timeinvariant case in Section 3.1. Introduce the identity

$$
\begin{aligned}
1= & A_{t+k}\left(q^{-1}\right)+f_{1} q^{-1} A_{t+k-1}\left(q^{-1}\right)+\ldots \\
& +f_{k-1} q^{-k+1} A_{t+1}\left(q^{-1}\right)+q^{-k} G_{t, k}\left(q^{-1}\right)
\end{aligned}
$$

which can be interpreted as the time-variant counterpart of (11). The coefficients of $F_{t, k}$ and $G_{t, k}$ can 
be determined using repeated polynomial division. For instance, for $f_{1}$ we have

$$
\frac{q\left(1-A_{t+k}\left(q^{-1}\right)\right)}{A_{t+k-1}\left(q^{-1}\right)}=f_{1}+\frac{R_{1}\left(q^{-1}\right)}{A_{t+k-1}\left(q^{-1}\right)} .
$$

By applying the same procedure on the remainder terms $R_{i}\left(q^{-1}\right)$, the rest of the coefficients of $F_{t, k}\left(q^{-1}\right)$ and $G_{t, k}\left(q^{-1}\right)$ can be determined. The corresponding output predictor is given by

$$
\begin{gathered}
\hat{y}(t+k)=\left(B_{t+k}\left(q^{-1}\right)+f_{1} q^{-1} B_{t+k-1}\left(q^{-1}\right)+\ldots\right. \\
\left.+f_{k-1} q^{-k+1} B_{t+1}\left(q^{-1}\right)\right) u(t+k-1) \\
+G_{t, k}\left(q^{-1}\right) y(t)+F_{t, k}\left(q^{-1}\right) \alpha_{t+k} . \quad
\end{gathered}
$$

As in (15) this expression can be partitioned in one part that depends on future control moves and one part that depends on past measured data only;

$$
\hat{y}(t+k)=S_{t, k}\left(q^{-1}\right) u(t+k-1)+\bar{y}(t+k) .
$$

Hence the output predictions can be expressed similar to (17), and the control sequence can be optimized analogously to the derivation in Section 3.1.

\subsection{General Numerical Optimiza- tion}

The most general solution to the nonlinear MPC problem is perhaps brute force optimization of the criterion (7) using nonlinear programming methods. That is, the optimal control sequence $\mathbf{u}$ is determined using a numerical optimization routine, that in each iteration simulates the system given the current value of the control sequence (that is, computes the $N$-step-ahead prediction), and then updates it in a direction such that the value of the objective function $J$ decreases.

It is known that more accurate results could be obtained if the optimization routine is provided with gradient information. From (20) we have that

$$
\nabla J=2 \mathbf{S}^{T} \mathbf{Q}_{\mathbf{e}}(\hat{\mathbf{y}}-\mathbf{r})+2 \mathbf{Q}_{\mathbf{u}} \mathbf{u}+2 \mathbf{D}^{T} \mathbf{Q}_{\boldsymbol{\Delta} \mathbf{u}} \boldsymbol{\Delta} \mathbf{u} .
$$

The gradient can thus be estimated from the local models obtained along the simulated trajectory.

A severe problem with the general optimization formulation, though, is that the optimization typically will be very complex and time-consuming for large control horizons $N_{u}$. We will most likely also get the problem with local minima.

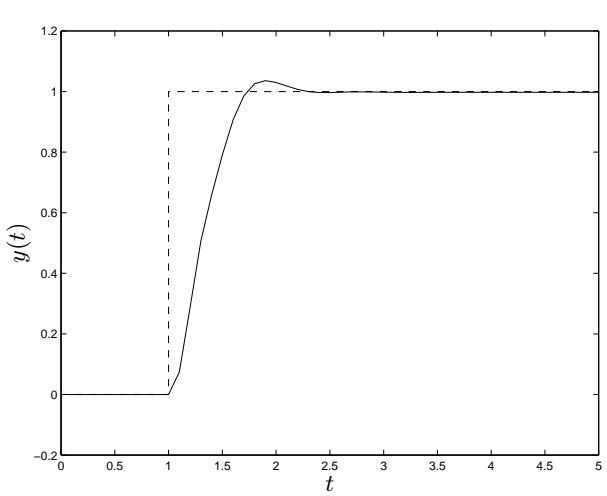

(a)

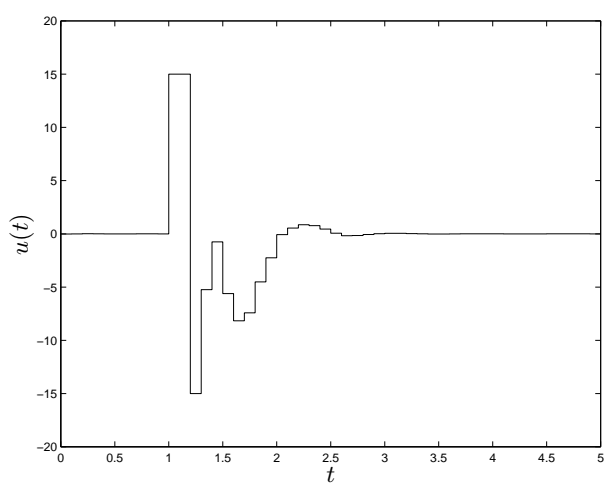

(b)

Figure 2: Step response experiment using the predictive controller of Section 3.1. (a) Reference signal (dashed) and system output (solid). (b) Control signal.

\section{A Simple Example}

To illustrate the proposed methods in simulations we will consider the nonlinear system

$$
\ddot{y}(t)(1+|y(t)|)=u(t) .
$$

It has the property that the gain of system decreases as the magnitude of the output signal increases.

The open-loop system is unstable, so a database consisting of 2000 output and regressor pairs $(y(k), \varphi(k))$ was built-up during a closed-loop experiment using a proportional controller, $u(t)=$ $K(y(t)-r(t))$, and a Gaussian reference signal $r(t)$. The sampling interval was selected as $T_{s}=0.1 \mathrm{sec}-$ onds. A simulation using this database and the predictive controller of Section 3.1 with parameter values $N=10, N_{u}=7, Q_{e}=1, Q_{u}=0$ and $Q_{\Delta u}=0.001$, and assuming that the control mag- 
nitude is limited according to

$$
|u(t+k)| \leq 15, \quad k=0, \ldots, N_{u}-1
$$

is shown in Figure 2.

A simulation using the same parameter values but the controller of Section 3.2 is shown in Figure 3. We see that the second controller gives a much better result. Since this controller uses linear models of the plant along the predicted future trajectory, it is aware of that the gain of the system will decrease as the output magnitude increases. Therefore it is more restrictive in its use of control energy as the output approaches the setpoint.

\section{Conclusions}

We have here presented the method of modelfree predictive control, which combines the idea of model-on-demand with established and well-known MPC techniques. The method is model-free in the sense that no global model of the process dynamics is required. Instead it relies upon an on-line estimation scheme that uses process data stored in a database. The only global model consideration is the configuration of the regression vector. Since the controller part of the algorithm is based on wellknown techniques, standard MPC tuning guidelines apply.

\section{References}

K.J. Åström and B. Wittenmark. Adaptive Control. Addison-Wesley, second edition, 1995.

D.W. Clarke, C. Mohtadi, and P.S. Tuffs. Generalized predictive control - I. The basic algorithm. Automatica, 23:137-148, 1987a.

D.W. Clarke, C. Mohtadi, and P.S. Tuffs. Generalized predictive control - II. Extensions and interpretations. Automatica, 23:149-160, 1987b.

W.S. Cleveland and S.J. Devlin. Locally weighted regression: an approach to regression analysis by local fitting. Journal of the American Statistical Association, 83:596-610, 1988.

J. Fan and I. Gijbels. Local Polynomial Modelling and Its Applications. Chapman \& Hall, 1996.

C.G. García, D.M. Prett, and M. Morari. Model predictive control: Theory and practice - a survey. Automatica, 25(3):335-348, 1989.

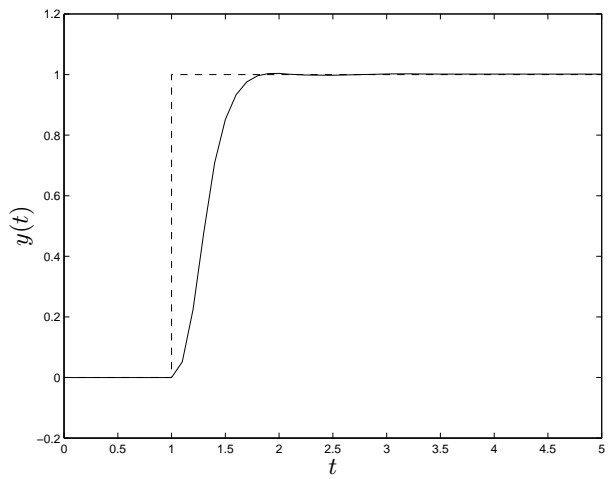

(a)

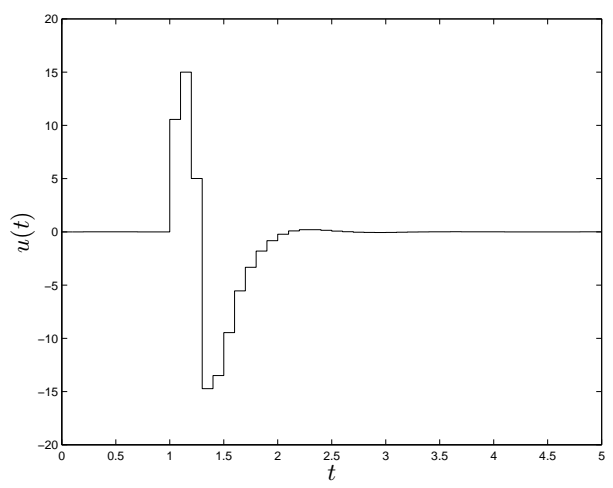

(b)

Figure 3: Step response experiment using the predictive controller of Section 3.2. (a) Reference signal (dashed) and system output (solid). (b) Control signal.

G. Gattu and E. Zafiriou. Nonlinear quadratic dynamic matrix control with state estimation. Ind. Eng. Chem. Res., 31(4):1096-1104, 1992.

C.R. Loader. Old faithful erupts: Bandwidth selection reviewed. Technical report, AT\&T Bell Laboratories, 1995.

J. Löfberg. Notes on NLPC. Technical report, Dept of EE, Linköping University, SE-581 83 Linköping, Sweden, 1998. To Appear.

E.S. Meadows and J.B. Rawlings. Model predictive control. In M.A. Henson and D.E. Seborg, editors, Nonlinear Process Control, chapter 5. Prentice Hall, 1997.

A. Stenman. Just-in-Time Models with Applications to Dynamical Systems. Licentiate thesis LIU-TEK-LIC-1997:02, Department of Electri- 
cal Engineering, Linköping University, S-581 83 Linköping, Sweden, 1997.

A. Stenman, F. Gustafsson, and L. Ljung. Just in time models for dynamical systems. In Proceedings of the 35th IEEE Conference on Decision and Control, Kobe, Japan, pages 1115-1120, 1996.

A. Stenman, A.V. Nazin, and F. Gustafsson. Asymptotic properties of Just-in-Time models. In Y. Sawaragi and S. Sagara, editors, Preprints of the 11th IFAC Symposium on System Identification, Kitakyushu, Japan, pages 1249-1254, 1997.

M.P. Wand and M.C. Jones. Kernel Smoothing. Chapman \& Hall, 1995. 